\title{
Análise da decomposição da desigualdade de renda no Estado do Ceará
}

\section{Analysis of the decomposition income inequality in the State of Ceará}

\author{
Gabriel Alves de Sampaio Morais ${ }^{1}$ \\ Jair Andrade Araujo ${ }^{2}$ \\ Denise Moreira Araújo de Santana ${ }^{3}$
}

\section{Resumo}

Os governos, a partir das políticas de desenvolvimento, buscam aumentar o bemestar da população. Dentre os vários objetivos, o combate à desigualdade de renda constitui uma prioridade. O presente artigo objetiva analisar a contribuição das diferentes parcelas do rendimento domiciliar per capita no estado do Ceará, entre 2004 e 2012, para identificar quais foram aquelas que mais contribuíram para a redução da desigualdade nos últimos anos. Os resultados permitem concluir que ocorreu uma redução da desigualdade de renda no estado, passando de 0,5708 em 2004 para 0,5203 em 2012. A análise da desigualdade conclui, ainda, que o efeito-concentração é predominante, ou seja, a diminuição da concentração de cada parcela de renda foi o principal fator para a redução da desigualdade.

Palavras-chave: Desigualdade de Renda. Coeficiente de Gini. Decomposição.

\section{Abstract}

The governments, from development policies seek to increase the welfare of the population. Among several objectives, combating income inequality is a priority. This article aims to analyze the contribution of different portions of household income per capita in the state of Ceará, between 2004 and 2012, to identify which were the ones that contributed most to the reduction of inequality in recent years. The results

\footnotetext{
1 Graduado em Análise e Desenvolvimento de Sistemas. Mestrando em Economia Rural (MAER/ UFC). Rua Dr. Floro Bartolomeu, 862 - Centro. Juazeiro do Norte, CE - Brasil. E-mail: Gabriel_ morais@yahoo.com.br

2 Doutor em Economia. Professor do Curso de Pós-Graduação em Economia Rural (MAER / UFC). Av. Mister Hull, Bloco 826 - Campus do Pici/UFC. Fortaleza/Ceará. CEP: 60.356-000. E-mail: jairandrade@ufc.br

3 Mestre em Economia. Funcionária da SEFAZ-CE. E-mail: denamoreira@hotmail.com
} 
showed that there was a reduction in income inequality in the State from 0.5708 in 2004 to 0.5203 in 2012. The analysis of inequality also allows to concludes that the concentration-effect is predominant, in other words, a decrease in concentration of each share of income was the main factor for the reduction of inequality.

Keywords: Income Inequality. Gini Coefficient. Decomposition.

\section{Introdução}

Os governos, a partir de políticas de desenvolvimento, buscam aumentar o bem-estar da população. Dentre os vários objetivos de suas políticas, o combate à desigualdade de renda constitui uma prioridade em muitos casos. No entanto, deve-se lembrar de que, apesar do aumento da capacidade em gerar riqueza de certas economias, a má distribuição da riqueza tem se mostrado como um fenômeno persistente.

A literatura sobre o assunto tem evidenciado que, mesmo nos casos mais bem sucedidos de crescimento econômico, a expansão do Produto Interno Bruto (PIB) não necessariamente tem beneficiado todos os indivíduos de uma determinada sociedade. Na verdade, tem-se verificado que até mesmo os países ricos têm encontrado dificuldades para eliminar redutos remanescentes de pobreza, observando-se o crescimento das desigualdades de renda (ROCHA, 2006).

Cabe salientar que a desigualdade de renda nos países em desenvolvimento é um problema ainda mais grave do que nos países desenvolvidos. Esse fato é reconhecido pela literatura internacional e tem-se mostrado evidente desde o último século. Isso fica claro quando se compara qualquer indicador de desigualdade tradicional, como o coeficiente de Gini (ARAÚJO; MORAIS, 2013).

No Brasil, o tema da desigualdade de renda tem sido frequentemente abordado por estudiosos em geral, originando diversas explicações peculiares para o assunto. Por se tratar de um país de dimensões continentais, o Brasil se configura como um território de sérios contrastes sociais, econômicos e com uma das mais elevadas taxas de desigualdade da América, além de ser constantemente 
associado a elevados índices de pobreza. Em geral, os estudos sobre desigualdade no país têm mostrado a existência histórica de profundos desníveis sociais nas condições de vida, principalmente de renda, entre os residentes das regiões brasileiras.

Apesar da diversidade de estudos encontrada na literatura, ainda há dificuldades para se encontrar explicações quanto aos fatores determinantes da desigualdade de renda e, algumas vezes, constituemse como uma fonte de controvérsias, justificando a realização de novos estudos. O desenvolvimento de estudos dessa natureza, voltados para a realidade brasileira, continua sendo importante, pois o Brasil é um país que apresenta enormes disparidades de renda. Além disso, as informações geradas podem auxiliar a formulação de políticas públicas capazes de promover um maior crescimento econômico, com maior equidade (FOCHEZATTO, 2011).

Há várias estatísticas que têm servido para revelar a magnitude dessas desigualdades, como a percentagem da população analfabeta, de pessoas vivendo abaixo da linha da pobreza e de residentes em domicílios considerados precários, segundo o IBGE. Diversas são as explicações para esse fenômeno, sendo as principais delas ligadas ao nível de capital humano entre as regiões, pois os indivíduos residentes nos estados ou municípios mais pobres têm menos educação formal (BARROS, 2013).

Nesse contexto, o objetivo deste estudo é analisar a contribuição das diferentes parcelas do rendimento domiciliar per capita no Ceará, entre 2004 e 2012, para identificar quais foram aquelas que mais auxiliaram na redução da desigualdade nos últimos anos. Além disso, faz-se uma análise da decomposição da desigualdade no estado do Ceará. A principal interrogação que se coloca dessa questão diz respeito a quais são os componentes de renda que influenciam a diminuição da desigualdade no Ceará. Salienta-se que o artigo trata exclusivamente dos aspectos relacionados à renda e de como sua evolução contribui para a redução das desigualdades. 
Para alcançar o objetivo proposto, adotou-se a metodologia de decomposição do índice de Gini, detalhada por Hoffmann (2007), utilizando as informações das PNADs. As informações geradas serviram de subsídio para compreender quais parcelas de renda influenciaram as alterações no índice de concentração de renda no estado durante o período selecionado. Ademais, realizou-se uma análise das mudanças na desigualdade.

Além desta introdução, este artigo é composto por seis seções. A segunda seção se trata de um breve histórico da desigualdade brasileira, alem de mostrar a sua relação com os principais determinantes. A seção seguinte define e discute a base de dados. A quarta seção apresenta o modelo adotado para a decomposição do índice de Gini. Finalmente, a quinta e sexta seções apresentam a análise dos resultados e as conclusões, respectivamente.

\section{Trajetória da desigualdade de renda no Brasil}

Esta seção apresenta uma breve trajetória da desigualdade de renda no Brasil durante as últimas décadas e seus principais determinantes.

Em geral, a literatura nacional registra um aumento significativo da desigualdade de renda brasileira na década de 1970, mantendose elevada nos períodos seguintes, até a metade da década de 1990. Entre uma das causas para tal, menciona-se o processo hiperinacionário brasileiro presente nesse período, afetando diretamente os rendimentos dos mais pobres. Essa situação começou a se modificar após o Plano Real, quando o país passou a desfrutar de relativa estabilidade macroeconômica, resultando em uma crescente queda na desigualdade de renda dos brasileiros (ARAÚJO, 2009).

Considerando os índices de Gini e Theil calculados para o período de 1976 a 2004, verificou-se uma diminuição da concentração de renda, a saber: (i) no período de 1976 a 1981, o coeficiente de Gini caiu em aproximadamente $7 \%$ e o índice de Theil, em 22\%; (ii) no período de 
1989 a 1992, o coeficiente de Gini se reduziu em 8\% e o índice de Theil, em 22\%; e (iii) no período de 2001 a 2004, o coeficiente de Gini caiu 4\% e o índice de Theil, 9\% (BARROS et al., 2006).

Analisando dados da PNAD, constata-se que a redução na desigualdade de renda no Brasil vem caindo continuamente desde 2001. Considerando o período de 2001 a 2011, por exemplo, a renda per capita dos $10 \%$ mais ricos cresceu $16,6 \%$ em termos acumulados. Por outro lado, a renda dos $10 \%$ mais pobres expandiu $91,2 \%$ no período analisado. Vale destacar que, conforme os dados da PNAD em 2011, o país obteve seu menor nível de desigualdade de renda desde os registros nacionais, iniciados em 1960. Entretanto, o Brasil continuou na lista dos 15 maiores do mundo em desigualdade de renda (IPEA, 2012).

Em geral, a literatura especializada no assunto apresenta um consenso de que está ocorrendo uma desconcentração da renda em favor dos mais pobres (HOFFMANN, 2007).

Analisando a Tabela 1, evidencia-se o declínio da desigualdade de renda brasileira no período de 2002 a 2011. As informações se referem ao índice de Gini, à razão entre a renda apropriada pelos $10 \%$ mais ricos e os $40 \%$ mais pobres (40-10+), à razão entre a renda apropriada pelos $20 \%$ mais ricos e os $20 \%$ mais pobres (20-20+), à razão entre a renda apropriada pelos $10 \%$ mais ricos e os $10 \%$ mais pobres (10-10+).

Sabe-se que o índice de Gini mede o grau de desigualdade existente na distribuição de indivíduos segundo a renda domiciliar per capita, para a economia brasileira. Conforme a Tabela 1, esse indicador apontou uma queda contínua de 0,5826 (em 2002) para 0,5241 (em 2011), significando uma redução de aproximadamente 5,8\%.

Além disso, a Tabela 1 mostra que a renda apropriada pelos 10\% mais ricos no Brasil representava 21,1 vezes a renda apropriada pelos $40 \%$ mais pobres em 2002. No ano de 2011, por exemplo, os ricos ganhavam 14,9 vezes o total de indivíduos pobres. Percebe-se, inclusive, que os indicadores (10-10+) e (20-20+) apresentaram queda significativa durante o período de 2002 a 2011 - principalmente o primeiro indicador, 
que passou de 23,00 em 2002 para 16,41 em 2011. Finalmente, a última coluna evidencia que a razão entre a renda apropriada pelos $10 \%$ mais ricos e os $10 \%$ mais pobres (10-10+) também caiu de forma contínua, passando de 50,44 (em 2002) para 36,37 (em 2011), ou seja, apresentando uma diferente de 14,07 pontos percentuais ao longo do período analisado.

Tabela 1: Indicadores de desigualdade da renda no Brasil ${ }^{1}$ em 2002, 2004-2011

\begin{tabular}{ccccc}
\hline Anos & Gini & $\mathbf{4 0 - 1 0 +}$ & $\mathbf{2 0 - 2 0 +}$ & $\mathbf{1 0 - 1 0 +}$ \\
\hline 2002 & 0,5826 & 21,18 & 23,00 & 50,44 \\
2004 & 0,5660 & 19,05 & 21,02 & 45,17 \\
2005 & 0,5637 & 18,83 & 20,38 & 44,42 \\
2006 & 0,5574 & 18,12 & 19,76 & 43,54 \\
2007 & 0,5482 & 17,37 & 18,85 & 41,46 \\
2008 & 0,5392 & 16,30 & 17,76 & 34,73 \\
2009 & 0,5354 & 16,20 & 17,49 & 38,38 \\
2011 & 0,5241 & 14,96 & 16,41 & 36,37 \\
\hline
\end{tabular}

Fonte: elaborado a partir dos microdados da PNAD (IBGE, 2002 a 2011)

Nota ${ }^{1}$ : Exclusive a área rural da antiga região Norte em 2002.

Em linhas gerais, os dados apresentados para o período de 2002 a 2011 revelam, de forma consistente, a ocorrência de uma redução contínua na desigualdade de renda no Brasil. Vale destacar que, apesar dos avanços obtidos no país, estudos recentes ainda chamam a atenção para a histórica concentração de renda da sociedade brasileira ao longo de décadas.

Na sequência, apresenta-se a Tabela 2, a qual mostra a evolução temporal da desigualdade de renda no Brasil durante o ano de 2002 e de 2004 a 2011. Verifica-se, então, que a concentração de renda é persistente. Os indivíduos que correspondem à parcela dos $20 \%$ mais ricos da população brasileira se apropriam de uma renda média 22 
superior à dos $20 \%$ mais pobres em 2004 e 16 vezes superior à dos 20\% mais pobres em 2011.

Além disso, a evolução temporal da desigualdade de renda no período em questão mostra que os indivíduos que se encontram entre os $10 \%$ mais ricos da população brasileira detêm aproximadamente $41 \%$ do total de renda das famílias. Por outro lado, os $50 \%$ mais pobres da população possuem pouco mais de $16 \%$ da renda, enquanto o grupo das pessoas $20 \%$ mais pobres se apropria somente de cerca de $3,48 \%$ do total da renda em 2011. Ressalta-se, inclusive que, no período analisado, os indivíduos do grupo composto pelos $10 \%$ mais ricos continuam se apropriando de uma parcela da renda superior à apropriada por metade de toda a população do Brasil.

Os dados do exercício empírico no ano de 2002 e de 2004 a 2011 confirmam a natureza concentradora de renda da sociedade brasileira. Apesar dos avanços alcançados, a desigualdade de renda no país não foi erradicada, constatando-se a persistência dos desníveis sociais nas condições de vida.

Tabela 2: Evolução temporal da desigualdade de renda no Brasil $^{2}$ em 2002, 2004-2011

\begin{tabular}{|c|c|c|c|c|c|c|}
\hline \multirow[b]{2}{*}{ Ano } & \multicolumn{6}{|c|}{ Porcentagem da renda apropriada pelas pessoas } \\
\hline & $\begin{array}{c}10 \% \text { mais } \\
\text { pobres }\end{array}$ & $\begin{array}{c}20 \% \text { mais } \\
\text { pobres }\end{array}$ & $\begin{array}{c}40 \% \text { mais } \\
\text { pobres }\end{array}$ & $\begin{array}{c}50 \% \text { mais } \\
\text { pobres }\end{array}$ & $\begin{array}{c}20 \% \text { mais } \\
\text { ricos }\end{array}$ & $\begin{array}{c}10 \% \text { mais } \\
\text { ricos }\end{array}$ \\
\hline 2002 & 0,93 & 2,73 & 9,23 & 13,36 & 62,70 & 46,51 \\
\hline 2004 & 0,99 & 2,96 & 9,61 & 14,21 & 60,46 & 44,93 \\
\hline 2005 & 1,01 & 3,02 & 9,56 & 14,61 & 60,62 & 44,94 \\
\hline 2006 & 1,06 & 3,10 & 9,85 & 14,78 & 60,36 & 44,25 \\
\hline 2007 & 1,06 & 3,21 & 10,22 & 15,21 & 59,22 & 43,18 \\
\hline 2008 & 1,20 & 3,29 & 10,73 & 15,80 & 58,73 & 42.86 \\
\hline 2009 & 1,11 & 3,32 & 10,81 & 16,02 & 58,19 & 42.10 \\
\hline 2011 & 1,15 & 3,48 & 11,15 & 16,62 & 57.06 & 41.30 \\
\hline
\end{tabular}

Fonte: elaborado a partir dos microdados da PNAD (IBGE, 2002 a 2011)

Nota2: Exclusive a área rural da antiga região Norte em 2002. 
Em termos gerais, pode-se afirmar que houve uma queda na concentração de renda no país a partir da década de 1990. No entanto, a trajetória dessa redução ocorreu de forma diferenciada nas regiões brasileiras (IPECE, 2011). A Tabela 3 mostra o índice de Gini obtidos para o Brasil e as regiões nos anos de 2000 e 2010, bem como a posição relativa em termos de nível e variação da desigualdade no período

Tabela 3: Índice de Gini da distribuição do rendimento nominal mensal dos domicílios particulares permanentes - Brasil e Regiões - 2000/2010

\begin{tabular}{l|cc|cc|cc}
\hline Brasil e Regiões & $\mathbf{2 0 0 0}$ & Rank & $\mathbf{2 0 1 0}$ & Rank & Variação (\%) & Rank \\
\hline Brasil & 0,597 & - & 0,536 & - & $-10,218$ & - \\
Norte & 0,598 & 3 & 0,543 & 3 & $-9,197$ & 5 \\
Nordeste & 0,612 & 2 & 0,555 & 1 & $-9,314$ & 4 \\
Sudeste & 0,575 & 4 & 0,517 & 4 & $-10,087$ & 3 \\
Sul & 0,564 & 5 & 0,480 & 5 & $-14,894$ & 1 \\
Centro-Oeste & 0,621 & 1 & 0,547 & 2 & $-11,916$ & 2 \\
\hline
\end{tabular}

Fonte: IPECE (2011, s.p.)

Observa-se que a região Sul se caracteriza por apresentar o menor nível de desigualdade do país ( $2^{a}$ e $3^{a}$ colunas da Tabela 3 ), produzindo, inclusive, a maior queda no índice de Gini durante o período considerado (última coluna da Tabela 3). Já a região Centro-Oeste se destacou em 2000 como a região com a pior concentração de renda do país, passando para a segunda posição em 2010. Vale ressaltar que a referida região expressou o segundo maior avanço na igualdade de rendimentos. No caso do Nordeste, apesar de ter registrado uma redução na concentração de renda durante o período, a região apresentou a segunda pior concentração de renda em 2000, passando ao posto de região mais desigual do país em 2010.

Além disso, verifica-se que em todos os estados brasileiros houve uma queda na desigualdade de renda. A Tabela 4 mostra o índice de Gini dos estados brasileiros nos anos de 2000 e 2010. Nesse período, Santa Catarina foi classificado como o estado menos desigual. Por outro lado, em 2000, o estado considerado mais desigual foi o Ceará e, em 2010, o 
Distrito Federal. Com relação ao Ceará, ressalta-se que o estado esteve entre os dez estados que mais melhoraram sua distribuição de renda no período. Ademais, destacou-se em relação aos demais estados nordestinos, sendo o que mais reduziu a desigualdade durante o período em análise ${ }^{4}$. Sergipe foi o estado que obteve pior resultado.

Tabela 4: Índice de Gini da distribuição do rendimento nominal mensal dos domicílios particulares permanentes - Unidades da Federação $2000 / 2010$

\begin{tabular}{l|cc|cc|cc}
\hline Unidades da Federação & $\mathbf{2 0 0 0}$ & Rank & $\mathbf{2 0 1 0}$ & Rank & Variação (\%) & Rank \\
\hline Rondônia & 0,575 & 21 & 0.505 & 21 & -12.174 & 8 \\
Acre & 0,590 & 16 & 0.550 & 12 & -6.780 & 22 \\
Amazonas & 0,600 & 14 & 0.557 & 5 & -7.167 & 21 \\
Roraima & 0.560 & 26 & 0.553 & 8 & -1.250 & 26 \\
Pará & 0.602 & 10 & 0.539 & 16 & -10.465 & 13 \\
Amapá & 0.579 & 19 & 0.547 & 13 & -5.527 & 25 \\
Tocantis & 0.604 & 9 & 0.540 & 15 & -10.596 & 11 \\
Maranhão & 0.609 & 6 & 0.547 & 13 & -10.181 & 14 \\
Piauí & 0.621 & 4 & 0.560 & 3 & -9.823 & 17 \\
Ceará & 0.626 & 1 & 0.556 & 7 & -11.182 & 10 \\
Rio Grande do Norte & 0.605 & 8 & 0.552 & 10 & -8.76 & 19 \\
Paraíba & 0.601 & 11 & 0.553 & 8 & -7.987 & 20 \\
Pernambuco & 0.622 & 3 & 0.559 & 4 & -10.129 & 16 \\
Alagoas & 0.623 & 2 & 0.557 & 5 & -10.594 & 12 \\
Sergipe & 0.568 & 23 & 0.563 & 2 & -0.880 & 27 \\
Bahia & 0.611 & 5 & 0.551 & 11 & -9.820 & 18 \\
Minas Gerais & 0.584 & 17 & 0.508 & 20 & -13.014 & 6 \\
Espírito Santo & 0.579 & 19 & 0.514 & 18 & -11.226 & 9 \\
Rio de Janeiro & 0.574 & 22 & 0.538 & 17 & -6.272 & 23 \\
São Paulo & 0.561 & 24 & 0.504 & 23 & -10.16 & 15 \\
Paraná & 0.580 & 18 & 0.488 & 26 & -15.862 & 3 \\
Santa Catarina & 0.540 & 27 & 0.448 & 27 & -17.037 & 1 \\
Rio Grande do Sul & 0.561 & 24 & 0.490 & 25 & -12.656 & 7 \\
Mato Grosso do Sul & 0.601 & 11 & 0.513 & 19 & -14.642 & 5 \\
Mato Grosso & 0.601 & 11 & 0.499 & 24 & -16.972 & 2 \\
Goiás & 0.598 & 15 & 0.505 & 21 & -15.552 & 4 \\
Distrito Federal & 0.607 & 7 & 0.573 & 1 & -5.601 & 24 \\
\hline
\end{tabular}

Fonte: IPECE (2011, s.p.)

4 A distribuição de renda no referido estado será discutida mais detalhadamente no desenvolvimento deste trabalho. 
A próxima subseção discutirá os principais determinantes da recente queda na desigualdade de renda no Brasil.

\subsection{Principais determinantes na redução da desigualdade de renda no Brasil}

Como foi dito anteriormente, a literatura apresenta uma diversidade de pesquisas que buscam investigar a evolução da desigualdade de renda no Brasil. Apesar dos avanços alcançados nesse campo de estudo, ainda não há consenso sobre quais dos elementos formadores de renda são os que mais contribuem para a redução dessa desigualdade. As principais hipóteses parecem recair principalmente sobre os programas oficiais de transferência de renda, a educação e o mercado de trabalho.

Segundo o IPEA (2012), a redistribuição de renda representa um dos fenômenos econômicos e sociais mais importantes ocorridos na última década no Brasil. Em linhas gerais, um conjunto de fatores pode ajudar a explicar essa transformação, como o controle da hiperinflação a partir da década de 1980 e, principalmente, em meados da década de 1990, a estabilidade econômica e a criação de políticas sociais de transferência de renda condicionadas (Programa de Erradicação do Trabalho Infantil, Bolsa Escola, Bolsa Alimentação, Cartão Alimentação e Bolsa Família).

Surgiram, então, diversas pesquisas que buscavam mostrar a relação entre a redução das desigualdades de renda e a implementação de programas sociais do governo. No entanto, em geral, os trabalhos desenvolvidos mostraram que existem muitas controvérsias quanto à eficácia desse tipo de política. Algumas pesquisas apontaram para a eficácia dos programas sociais. Por outro lado, outros estudos mostraram conclusões contrárias, chegando a afirmar que as transferências governamentais desencorajam os indivíduos pobres a procurarem emprego, tornando-os dependentes das transferências contínuas do governo (ARAÚJO; MORAIS, 2013).

Segundo o IPEA (2012), por exemplo, a redução de desigualdade observada na década passada pode ser decomposta pelas diversas 
fontes de renda captadas pela PNAD, a saber: trabalho (58\%); previdência (19\%); Bolsa Família (13\%); benefício de prestação continuada ${ }^{5}(4 \%)$; e outras rendas, como aluguéis e juros (6\%). Em outras palavras, a maior parte da queda da desigualdade se deveu ao efeito da expansão trabalhista observada, o que confere sustentabilidade ao processo redistributivo assumido. A pesquisa mostrou, ainda, que sem as políticas redistributivas patrocinadas pelo Estado brasileiro, a desigualdade teria caído 36\% menos na década.

Soares (2006) destaca a política social do governo, baseando-se nos programas de transferência de renda, para a redução dos diferenciais de renda. $\mathrm{O}$ autor acrescentou seu estudo ao mostrar que a mudança no mercado de trabalho também contribui para a redução da desigualdade de renda brasileira.

Seguindo a mesma linha, Medeiros, Brito e Soares (2007) afirmaram que os programas de transferência de renda no Brasil contribuem para reduzir a pobreza e a desigualdade. Ressaltaram, ainda, que não há indicação de que as transferências afetem de modo substantivo a participação das famílias no mercado de trabalho. Verificaram inclusive que a participação no mercado de trabalho aumentou entre os beneficiários. Esses argumentos corroboram com os estudos de Barros et al. (2010), os quais afirmaram ter ocorrido uma redução da desigualdade motivada também pelas sucessivas reduções nos diferenciais de remuneração por nível educacional.

Araújo (2009), por sua vez, concluiu que as transferências de renda não afetam a dinâmica da desigualdade de renda no período de 1995 a 2009. Em seu estudo, o autor destaca a educação como sendo o principal determinante na redução da desigualdade de renda no Brasil, seguida por todos os trabalhos. Ademais, a pesquisa mostrou que a redução de impostos, por meio de política fiscal eficiente, também contribuiria para a redução das disparidades de renda no país.

$\mathrm{Na}$ mesma perspectiva, menciona-se o trabalho de Marinho, Linhares e Campelo (2007), os quais também concluíram que os programas de transferência de renda no Brasil não contribuem para 
reduzir a pobreza, além de chamarem atenção para o fato de que os beneficiários dos programas sociais, em sua maioria, não possuem incentivos para buscar outros meios para obter renda, tornando-se dependente desses programas.

Barros, Foguel e Ulyssea (2006) destacaram cinco determinantes que contribuíram para a redução da desigualdade de renda no Brasil recentemente: (i) características demográficas das famílias, havendo redução na desigualdade demográfica entre famílias ricas e pobres; (ii) programas de transferência de renda do governo brasileiro, compostos de três componentes (pensões e aposentadorias públicas, o beneficio de prestação continuada e o programa Bolsa Família) que contribuíram com cerca de 1/3 na diminuição da concentração de renda; (iii) remuneração de ativos, embora os autores não encontrem que esse componente tenha participação significativa na redução da desigualdade; (iv) acesso ao trabalho e participação no mercado de trabalho explicam 3\% da redução da desigualdade entre 2001 e 2004; (v) distribuição dos rendimentos provenientes do trabalho, influenciada por escolaridade, experiência do trabalhador, raça, sexo, entre outros fatores, explica cerca de $15 \%$ da queda da desigualdade de renda familiar per capita no Brasil.

Cabe salientar que o objetivo desta subseção não foi esgotar o assunto sobre os fatores determinantes na redução da desigualdade de renda no Brasil, mas mostrar a diversidade desses fatores apresentados na literatura e melhorar a compreensão desse fenômeno.

\subsection{Principais determinantes na redução da desigualdade de renda no Ceará}

A desigualdade de renda existente entre os estados e as regiões brasileiras, notadamente entre a região Sudeste e as regiões Norte e Nordeste, é tema presente na literatura econômica e objeto permanente de discussões entre acadêmicos, elaboradores de políticas governamentais e membros da classe política.

Utilizando o modelo econométrico de dados em painéis, Barreto, Neto e Tebaldi (2001) verificaram a relação entre desigualdade e 
crescimento para os estados do Nordeste no período de 1970 a 1999 . Os dados utilizados foram: o PIB per capita e o índice de Gini. Dentre os resultados obtidos, constataram que os nove estados nordestinos apresentaram um aumento na concentração de renda entre 1970 e 1991, e que o Ceará era o estado com renda mais concentrada entre os nordestinos em 1970, 1980 e 1991. No entanto, seis estados, incluindo o Ceará, indicaram uma reversão dessa tendência no ano de 1999. A concentração de renda gerou externalidades negativas para a economia do Nordeste e, consequentemente, prejudicou o crescimento do produto per capita no período analisado.

Neder e Silva (2004) desenvolveram aplicações de metodologias para a estimativa de índices de pobreza e de distribuição de renda em áreas rurais do Brasil com os dados das PNADs de 1995 e 2001. Dentre as principais conclusões, foi averiguada uma queda significativa na concentração de renda das áreas rurais de Tocantins, Piauí e Paraíba; nos estados de São Paulo e Paraná, constatou-se uma elevação significativa do valor do índice de Gini. Em termos de distribuição de renda rural, a região Nordeste apresenta dois padrões: os estados do Maranhão, Piauí, Ceará e Rio Grande do Norte com elevados índices de Gini, ao passo que os estados da Paraíba, Pernambuco, Alagoas, Sergipe têm valores significativamente mais baixos.

Penna e Neto (2009) confirmaram uma análise alternativa da hipótese de Kuznets (associação entre crescimento econômico e desigualdade de renda que se concretiza numa função com formato de um U invertido) para o Ceará, baseada na decomposição vetorial da renda, a partir dos dados do Atlas do Desenvolvimento Humano no Brasil (PNUD) para os anos de 1991 e 2000. Utilizando a metodologia das regressões quantílicas, perceberam que o índice de Gini apresentou um pior desempenho para quase todos os municípios do Ceará, revelando que o processo de concentração de renda se agravou para todo o estado. Os resultados indicaram que a redução das desigualdades deve ser mais eficiente se o crescimento econômico ocorrer por meio da geração de emprego e renda do que se for ocasionada pelo aumento das transferências governamentais. 
A partir de uma base de dados municipal, Marques (2010) investigou as diferenças no crescimento econômico e no grau de desigualdade de renda entre os estados da região Nordeste no período 2002-2006. A metodologia empregada baseou-se no teste Kruskal-Wallis e no teste $U$ de Wilcoxon-Mann-Whitney. Os resultados encontrados indicaram uma correlação linear inversa entre crescimento e desigualdade estatisticamente significativa nos municípios da Paraíba, Ceará e Alagoas. O caso de extrema desigualdade é representado pelos municípios situados nos estados de Sergipe e Ceará, que formam o grupo de municípios mais desiguais do Nordeste.

Salvato, Ferreira e Duarte (2010) buscaram identificar o quanto do diferencial de renda entre as regiões Nordeste e Sudeste e entre o estado do Ceará e São Paulo é explicado pelo diferencial de escolaridade da população. Usaram um modelo semiparamétrico para construir funções de densidade confractuais, reponderando os indivíduos da região/ estado base pela distribuição de educação, a ser comparada a partir das informações da PNAD de 1999. Dentre os principais resultados relevantes, verificou-se que: (i) a dispersão de renda é maior nas distribuições com menor média, ou seja, a desigualdade de renda, que é enorme em todas as regiões, é maior nas regiões mais pobres; (ii) entre $12 \%$ e $36 \%$ da diferença de renda do trabalho entre a região Nordeste e a região Sudeste e entre os estados de São Paulo e Ceará se deve às diferenças de escolaridade.

Com o intuito de analisar a desigualdade econômica entre a região metropolitana de Fortaleza e o restante do estado do Ceará, Pontes (2011) utilizou a decomposição de Oaxaca a partir dos dados das PNADs 1992, 2001 e 2009. Os resultados encontrados mostraram a redução da desigualdade, sendo possível intensificar esse processo por meio de políticas de incentivo à educação, dado que esse setor constitui um importante fator para a diferença entre os rendimentos regionais.

Miro, Suliano e Oliveira (2011) empregaram uma metodologia para avaliar a contribuição dos determinantes da renda familiar per capita (aspectos demográficos, a composição da renda familiar de acordo com 
suas fontes - renda do trabalho e renda não derivada do trabalho - e a taxa de ocupação no mercado de trabalho) na redução da desigualdade de renda para o estado do Ceará a partir dos dados das PNADs de 2003 a 2009. Constataram que o estado cearense apresentou elevadas reduções da desigualdade em decorrência do crescimento da renda dos menos favorecidos. Tal fato se deve ao papel das transferências, que compõem a renda não derivada do trabalho, responsáveis por quase $50 \%$ na redução da desigualdade. Outro resultado relevante foi a baixa contribuição do mercado de trabalho para a redução da desigualdade de renda no Ceará no período analisado.

Na Tabela 5, são apresentados os indicadores da desigualdade de renda no estado do Ceará de 2002 a 2011. Há o índice Gini para a Região Metropolitana de Fortaleza (RMF), zona urbana e rural. Além disso, mostra-se a razão entre a renda média dos 10 mais ricos e os $50 \%$ mais pobres (10-50+), e a razão entre a renda média dos $20 \%$ mais ricos e os $20 \%$ mais pobres.

Tabela 5: Indicadores de desigualdade de renda do estado do Ceará

\begin{tabular}{c|ccc|cc}
\hline Anos & RMF & Urbano & Rural & $10-50+$ & $20-20+$ \\
\hline 2002 & 0,601 & 0,505 & 0,487 & 17,9 & 23,2 \\
2003 & 0,586 & 0,498 & 0,741 & 15,9 & 20,9 \\
2004 & 0,600 & 0,497 & 0,408 & 16,3 & 19,3 \\
2005 & 0,579 & 0,528 & 0,491 & 16,8 & 21,7 \\
2006 & 0,557 & 0,493 & 0,437 & 14,1 & 18,7 \\
2007 & 0,550 & 0,504 & 0,477 & 14,2 & 19,5 \\
2008 & 0,557 & 0,480 & 0,441 & 13,4 & 16,1 \\
2009 & 0,555 & 0,488 & 0,444 & 13,8 & 18,3 \\
2011 & 0,522 & 0,521 & 0,459 & 13,3 & 18,9 \\
\hline
\end{tabular}

Fonte: elaborado a partir dos microdados da PNAD (IBGE, 2002 a 2011)

Verifica-se que a desigualdade de renda no estado do Ceará reduziu-se na RMF, principalmente entre 2002 e 2011, enquanto houve pequeno aumento da concentração de renda nas zonas urbana e rural entre 2009 e 2011. Segundo o IPECE (2011), a elevação da 
desigualdade de renda na zona urbana entre 2009 e 2011 se deve à taxa de crescimento da renda no último décul ter sido maior que a dos decis inferiores, enquanto na zona rural, além do crescimento da renda dos decis mais elevados, houve redução da renda dos três primeiros decis.

Ainda na Tabela 5, tem-se que a razão entre a renda média dos $10 \%$ mais ricos e os $50 \%$ mais pobres seguiu a mesma trajetória do índice de Gini. Já a razão entre a renda média dos $20 \%$ mais ricos e os $20 \%$ mais pobres passou de 23,2 em 2002 para 18,9 em 2011. De forma geral, pode-se afirmar que a renda é mais equitativa em 2011 se comparada à observada em 2002 no estado do Ceará.

\section{Metodologia}

Os dados utilizados na técnica de decomposição do índice de Gini foram retirados da Pesquisa Nacional por Amostragem Domiciliar (PNAD), publicada pelo Instituto Brasileiro de Geografia e Estatística (IBGE). A amostra é composta por informações de domicílios e indivíduos residentes no estado do Ceará no período de 2004 a 2012.

$\mathrm{Na}$ base de dados, cada indivíduo ou domicílio representa um determinado número de pessoas ou domicílios da população. As informações são fornecidas com peso ou fator de expansão de cada observação. Assim sendo, pondera-se cada observação pelo respectivo peso. Nesta pesquisa, considera-se sempre a última versão disponível para os pesos ou fator de expansão associados a cada indivíduo ou domicílio da amostra das PNADs, divulgados pelo IBGE.

A análise da desigualdade de renda é realizada a partir da renda domiciliar per capita, que compreende o quociente entre o rendimento domiciliar e o número de pessoas residentes, excluindo pensionistas, empregados domésticos e parentes de empregados domésticos. Essa variável considera a soma de todas as rendas disponíveis, tanto monetárias como não monetárias, de todos os indivíduos do domicílio.

Salienta-se que a renda domiciliar per capita é construída pelos seguintes componentes: renda do trabalho, que inclui a renda do trabalho 
principal, secundário e de outros trabalhos; rendimentos previdenciários (aposentadorias e pensões públicas e privadas); aluguéis; doações de outros domicílios; rendas de juros, dividendos, outros rendimentos e transferências de programas oficiais, como o Bolsa Família.

Assim sendo, a renda total é determinada pela soma da renda proveniente do trabalho e do não trabalho. Neste estudo, considera-se que o rendimento domiciliar é dividido em seis parcelas, a saber:

a) Rendimento do trabalho (TRA), que inclui a renda do trabalho, do trabalho secundário e de outros trabalhos, tanto monetário quando não monetário.

b) Aposentadoria e pensões públicas (AP1) correspondem a rendimentos de aposentadorias e pensões recebidas do instituto de previdência ou do governo federal.

c) Aposentadorias e pensões (AP2) são as aposentadorias e pensões privadas.

d) Aluguéis (ALU) compreendem os rendimentos de aluguel.

e) Abono e doações (DOA) são as doações recebidas de não morador e abono de permanência.

f) Outros rendimentos (OUT) incluem juros de caderneta de poupança e de outras aplicações, dividendos e rendimentos provenientes dos programas de transferência do governo por meio de programas sociais.

O procedimento de decomposição do índice de desigualdade utilizado segue os passos definidos em Hoffmann (2007).

Suponha que $x_{i}$ seja a renda da i-ésima pessoa em uma população formada por $\mathrm{n}$ indivíduos e que as rendas estão ordenadas de maneira que $\mathrm{x}_{1} \leq \mathrm{x}_{2} \leq \ldots \leq \mathrm{x}_{\mathrm{n}}$. Sendo a renda média dada por $\mu=\frac{1}{n} \sum_{i=1}^{n} x_{i}$ agregando as pessoas da mais pobre até a i-ésima posição na série, a proporção acumulada da população será $p_{i}=\frac{i}{n}$ e a respectiva proporção acumulada da renda será. $\Phi_{i}=\frac{1}{n \mu} \sum_{j=1}^{i} x_{j}$. Define-se o índice de Gini (G) por: 


$$
G=\frac{2}{n} \sum_{i=1}^{n-1}\left(p_{i}-\Phi_{i}\right)
$$

Esse indicador de desigualdade varia entre zero e um, e associa a área entre a curva de Lorenz e a linha de perfeita igualdade $\Phi=p$. A curva mostra como $\Phi_{\mathrm{i}}$ varia em função de .

Para o procedimento de decomposição do índice de Gini, inicialmente, admite-se que a renda das pessoas seja constituída por $\mathrm{k}$ parcelas, ou seja, $x_{i}=\sum_{h=1}^{k} x_{h i}$. Supõe-se que $x_{i}>0$ e $\beta$ a área entre a curva de Lorenz e o eixo das abscissas $p_{i}$. Assim sendo, o índice de Gini pode ser definido como:

$$
G=1-2 \beta
$$

Mantendo a ordenação das rendas, ou seja, $x_{1} \leq x_{2} \leq \ldots x_{n}$, a curva de concentração da parcela $x_{h i}$ mostra como a proporção acumulada dos

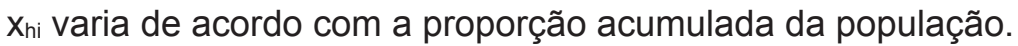

Define-se $\beta_{\mathrm{h}}$ como a área entre essa curva e o eixo das abscissas. A razão de concentração da parcela $x_{h i}$ é dada $C_{h}=1-\beta_{h}$ por, em que $-1<C_{h}<1$.

Sendo $\varphi_{h}$ a participação da h-ésima parcela total, o índice de Gini pode ser determinado por:

$$
G=\sum_{h=1}^{k} \varphi_{h} C_{h} \text {. }
$$

Portanto, o índice de Gini pode ser dividido em k componentes, correspondentes à $\mathrm{k}$ parcelas do rendimento domiciliar per capita. Hoffmann (2007) apresenta também a decomposição do indicador de desigualdade considerando dois anos distintos, indicados pelos índices 1 e 2.

$$
G_{1}=\sum_{h=1}^{k} \varphi_{1 h} C_{1 h} \quad(4) \text { e } \quad G_{2}=\sum_{h=1}^{k} \varphi_{2 h} C_{2 h}
$$

Assim, a variação no índice de Gini entre esse dois anos será:

$$
\Delta G=G_{2}-G_{1}=\sum_{h=1}^{k}\left(\varphi_{2 h} C_{2 h}-\varphi_{1 h} C_{1 h}\right)
$$


Somando e subtraindo e fatorando encontra-se:

$$
\Delta G=\sum_{h=1}^{k}\left(C_{2 h} \Delta \varphi_{h}+\varphi_{1 h} \Delta C_{h}\right)
$$

em que e. $\Delta \varphi_{h}=\varphi_{2 h}-\varphi_{1 h}$ e $\Delta C_{h}=C_{2 h}-C_{1 h}$.

Note que somando e subtraindo $\varphi_{2 \mathrm{~h}} \mathrm{C}_{1 \mathrm{~h}}$ dentro da expressão entre parênteses definida em ( 6 ), e fatorando, tem-se:

$$
\Delta G=\sum_{h=1}^{k}\left(C_{1 h} \Delta \varphi_{h}+\varphi_{2 h} \Delta C_{h}\right)
$$

As expressões definidas em ( 7 ) e ( 8 ) são duas maneiras possíveis de decompor $\Delta G$ No entanto, Hoffmann (2007) sugere utilizar a média aritmética das duas expressões para evitar a escolha arbitrária de uma delas da seguinte forma:

$$
\Delta G=\sum_{h=1}^{k}\left(C_{h}^{*} \Delta \varphi_{h}+\varphi_{h}^{*} \Delta C_{h}\right)
$$

em que $C_{h}^{*}=1 / 2\left(C_{1 h}+C_{2 h}\right)$ e $\varphi_{h}^{*}=\left(\varphi_{1 h}+\varphi_{2 h}\right)$.

Já a média dos índices de Gini nos dois anos considerados será:

$$
G^{*}=1 / 2\left(G_{1}+G_{2}\right)
$$

Como, $\sum \varphi_{2 h}=\sum \varphi_{1 h}=1$, verifica-se que:

$$
\sum_{h=1}^{k} G^{*} \Delta \varphi_{h}=G^{*} \sum_{h=1}^{k}\left(\varphi_{2 h}-\varphi_{1 h}\right)=0
$$

Assim sendo, a expressão ( 9 ) continua verdadeira ao subtrair a expressão (11) do segundo membro, obtendo:

$$
\Delta G=\sum_{h=1}^{k}\left[\left(C_{h}^{*}-G^{*}\right) \Delta \varphi_{h}+\varphi_{h}^{*} \Delta C_{h}\right]
$$

De forma matemática, tanto (9) como (12) são válidas. Porém, Hoffmann (2007) destaca que seria mais correto utilizar a expressão (12) na decomposição do índice de Gini. Verifica-se que o termo $\Delta G$ que representa variações no índice de Gini é dado pela soma de dois termos. Considerando a expressão (12) como a decomposição da mudança no índice de Gini, a contribuição total da h-ésima parcela do rendimento para essa mudança é:

$$
(\Delta G)_{h}=\left(C_{h}^{*}-G^{*}\right) \Delta \varphi_{h}+\varphi_{h}^{*} \Delta \mathrm{C}_{\mathrm{h}}
$$


Já a contribuição percentual será:

$$
S_{h}=\frac{100}{\Delta G}\left[\left(C_{h}^{*}-G^{*}\right) \Delta \varphi_{h}+\varphi_{h}^{*} \Delta \mathrm{C}_{\mathrm{h}}\right]
$$

Note que as expressões ( 13 ) e ( 14 ) podem distinguir um feito associado à mudança na composição do rendimento (efeito-composição) e um efeito associado à mudança nas razões de concentração (efeitoconcentração). Esses dois efeitos como porcentagem da mudança no índice de Gini serão $S_{\varphi h}=\frac{100}{\Delta G}\left[\left(C_{h}^{*}-G^{*}\right) \Delta \varphi_{h}\right]$ e $S_{c h}=\frac{100}{\Delta G}\left[\varphi_{h}^{*} \Delta \mathrm{C}_{\mathrm{h}}\right]$ respectivamente. Destaca-se que 0 efeito-composição total será $\sum_{h=1}^{k}\left(C_{h}^{*}-G^{*}\right) \Delta \varphi_{h}$ e o efeito-concentração da h-ésima parcela é $\varphi_{h}^{*} \Delta C_{h}$. Já o efeito-concentração da h-ésima parecela e total serão

$\varphi_{h}^{*} \Delta \mathrm{C}_{\mathrm{h}}$ e $\sum_{h=1}^{k} \varphi_{h}^{*} \Delta C_{h}$ e respectivamente. Ainda segundo Hoffmann (2007), o aumento da participação de uma parcela de renda contribui para aumentar ou diminuir o indicador de desigualdade de renda, conforme a razão de concentração dessa parcela seja maior ou menor do que o índice considerado, respectivamente.

\section{Resultados e discussão}

\subsection{Participações e razão de concentração dos componentes de renda no estado do Ceará}

Nesta seção, serão apresentados os resultados. Como explicado anteriormente, considera-se que a renda domiciliar percapita compreende a soma de seis parcelas, a saber: rendimentos de todos os trabalhos (TRA), aposentadorias e pensões públicas (AP1) aposentadorias e pensões privadas (AP2), aluguel (ALU), doações (DOA) e outros rendimentos (OUT). Entende-se que a desigualdade de cada um desses componentes de renda influencia na disparidade da renda domiciliar per capita, daí a importância em desagregar o coeficiente de Gini por seus componentes. 
Na Tabela 6, estão as participações ( $\varphi$ h) (de cada parcela na renda total. Verifica-se que, de 2004 a 2012, a participação de TRA diminui de $70,60 \%$ para $68,20 \%$. Porém, aumenta um pouco de 2009 para 2011 . Esse componente de renda é aquele que possui a maior participação na renda domiciliar per capita, seguido pela parcela da renda de aposentadoria e pensões oficiais (AP1), com uma média no período de aproximadamente $21 \%$. Resultados semelhantes foram encontrados por Araújo (2010), ao analisar a contribuição de várias parcelas do rendimento domiciliar para a desigualdade de renda brasileira.

Pode-se inferir que a queda da participação do TRA na renda total pode ser resultado das políticas públicas que contribuem para aumentar a participação da renda de outras fontes, a exemplo dos programas federais, como o Bolsa Família. Deve-se mencionar, ainda, que o envelhecimento da população e a elevação dos gastos públicos com aposentadorias contribuem para aumentar a participação desses segmentos na renda total. Assim sendo, tem-se o decréscimo de forma relativa da parcela oriunda exclusivamente da renda do trabalho na renda total.

Na dinâmica do mercado de trabalho e sua interação com a desigualdade, existe uma série de fatores que podem acarretar diferenças de salário entre os indivíduos que dele participam e, dessa forma, influenciar o nível de desigualdade de rendimentos. A esse respeito, Ramos (2007) destaca o seguinte: a) salários distintos ocasionados pelas diferenças não pecuniárias entre os postos de trabalhos na economia (como insalubridade, riscos de acidentes) estão associados à heterogeneidade dos postos de trabalho; b) diferenças de salários podem ser provenientes da heterogeneidade entre os trabalhadores; c) remuneração de forma distinta àqueles trabalhadores que são igualmente produtivos; e d) o mercado pode estar remunerando distintamente trabalhadores igualmente produtivos, com base em atributos não produtivos (como cor e sexo). Nesse caso, tem-se uma discriminação no mercado de trabalho. 
Já a participação de outros rendimentos (juros de caderneta de poupança e de outras aplicações, dividendos e rendimentos dos programas de transferência do governo) aumentou de 3,8\% para $5,8 \%$ durante todo o período 2004 a 2012. Essa mesma tendência foi encontrada por Araújo (2010), que analisou a decomposição do coeficiente de Gini no Brasil entre 2002 e 2008 e verificou também que a participação dessa parcela na renda domiciliar per capita cresceu nos últimos anos.

Tabela 6: Participação ( $\square$ h) de cada parcela na renda total no estado do Ceará

\begin{tabular}{l|l|l|l|l|l|l}
\hline Parcela & $\mathbf{2 0 0 4}$ & $\mathbf{2 0 0 6}$ & $\mathbf{2 0 0 8}$ & $\mathbf{2 0 0 9}$ & $\mathbf{2 0 1 1}$ & $\mathbf{2 0 1 2}$ \\
\hline TRA & 0,706 & 0,709 & 0,707 & 0,708 & 0,703 & 0,682 \\
\hline AP1 & 0,221 & 0,217 & 0,217 & 0,217 & 0,234 & 0,234 \\
\hline AP2 & 0,014 & 0,012 & 0,013 & 0,015 & 0,009 & 0,010 \\
\hline ALU & 0,009 & 0,007 & 0,013 & 0,011 & 0,005 & 0,010 \\
\hline DOA & 0,009 & 0,010 & 0,008 & 0,005 & 0,002 & 0,004 \\
\hline OUT & 0,038 & 0,043 & 0,039 & 0,042 & 0,044 & 0,058 \\
\hline Total & 1,000 & 1,000 & 1,000 & 1,000 & 1,000 & 1,000 \\
\hline
\end{tabular}

Fonte: elaborado a partir dos microdados da PNAD (IBGE, 2004 a 2012)

A participação de outros rendimentos ocupa o terceiro lugar na contribuição na renda total desde 2004 no estado do Ceará. A crescente participação desse componente indica um potencial efeito dos programas de transferência de renda do governo federal, como o Bolsa Família (Tabela 6).

Os rendimentos de aposentadorias e pensões privadas (AP2) elevaram a sua participação na renda de $2,12 \%$ para $2,34 \%$ entre 2004 e 2012, ou seja, durante todo o período, não ultrapassaram os $3 \%$. Os aluguéis (ALU), abonos e doações (DOA) tiveram uma pequena participação na renda total ao longo do período. Verifica-se que os rendimentos de abonos e doações não chegam a $2 \%$ (Tabela 6). Note ainda que, entre 2006 e 2007, houve uma diminuição na participação relativa de outros rendimentos (OUT). 
A Tabela 7 mostra as razões de concentração relativa ao índice de Gini da distribuição do rendimento domiciliar per capita no estado do Ceará entre 2004 a 2012. Na última linha, tem-se o valor do coeficiente de Gini em cada ano. Verifica-se uma diminuição na desigualdade de renda no estado, passando de 0,5708 em 2004 para 0,5203 em 2012. Esses resultados corroboraram com diversos autores, como Araújo (2010), que também encontrou tendência decrescente da desigualdade de renda brasileira.

Conforme a metodologia da decomposição do coeficiente de Gini, quando a existência do índice de concentração de um determinado componente é superior ao Gini total, esse componente possui um caráter regressivo, ou seja, contribui para ampliar a desigualdade de renda. Caso contrário, é classificado como progressivo.

As informações na Tabela 7 mostram que os valores das razões de concentração da aposentadoria e pensões oficiais (AP1) e das rendas de trabalho (TRA) se situam acima do valor do índice de Gini, o que vai de encontro aos resultados de Hoffmann (2007), que mostra que esses dois componentes contribuem para elevar o grau de desigualdade de distribuição de renda no Brasil. No entanto, ressalta-se a tendência decrescente na concentração dessas duas parcelas no período de 2004 a 2011.

As parcelas aposentadorias e pensões (API) e (AP2) são bastante concentradas. Porém, verifica-se uma relativa melhora em sua distribuição ao longo do período, ou seja, esses rendimentos, mesmo sem mudanças significativas em sua participação relativa, apresentaram um importante papel na distribuição de renda recente.

Ainda na Tabela 7, a renda de aposentadoria e pensões privadas (AP2) apresenta valores maiores que o índice de Gini entre 2004 e 2009, caracterizando-se um componente regressivo. Já a partir de 2011, a razão de concentração foi menor que o índice de concentração, tornando-se uma parcela um pouco progressiva. 
Tabela 7: Razões de concentração relativas ao índice de Gini da distribuição do rendimento domiciliar per capita no estado do Ceará.

\begin{tabular}{l|c|c|c|c|c|c}
\hline Parcela & $\mathbf{2 0 0 4}$ & $\mathbf{2 0 0 6}$ & $\mathbf{2 0 0 8}$ & $\mathbf{2 0 0 9}$ & $\mathbf{2 0 1 1}$ & $\mathbf{2 0 1 2}$ \\
\hline TRA & 0,573 & 0,561 & 0,546 & 0,555 & 0,560 & 0,557 \\
\hline AP1 & 0,647 & 0,594 & 0,593 & 0,595 & 0,566 & 0,566 \\
\hline AP2 & 0,640 & 0,586 & 0,539 & 0,550 & 0,498 & 0,491 \\
\hline ALU & 0,875 & 0,848 & 0,869 & 0,861 & 0,826 & 0,858 \\
\hline DOA & 0,398 & 0,421 & 0,392 & 0,326 & $-0,067$ & 0,202 \\
\hline OUT & 0,019 & $-0,048$ & $-0,087$ & $-0,079$ & $-0,095$ & $-0,136$ \\
\hline Total & 0,5708 & 0,5431 & 0,5346 & 0,5397 & 0,5319 & 0,5203 \\
\hline
\end{tabular}

Fonte: elaborado a partir dos microdados da PNAD (IBGE, 2004 a 2012)

A renda proveniente do aluguel (ALU) apresenta índice de concentração muito acima do índice de Gini. Assim sendo, pode ser classificada como bastante regressiva, corroborando com resultados de Araújo (2010). As informações na Tabela 6 mostram, ainda, que essa fonte de renda é a mais desigual dentre todas as analisadas, cujo índice de concentração foi em média de $85 \%$ ao longo do período, com uma leve tendência decrescente.

Com relação à renda proveniente de abono e doações (DOA), apesar de ser relativamente pouco concentrada, as razões de concentração na Tabela 4 apontam valores menores que o índice de Gini em todos os anos, ou seja, trata-se de uma distribuição progressiva, com tendência decrescente, passando de 39,80\% em 2004 para 20,2\% em 2012.

Observa-se, na Tabela 7, que a parcela outros rendimentos apresenta a principal mudança nas razões de concentração. Sem dúvida, caracteriza-se como uma extraordinária diminuição da razão concentração. Note-se que a razão de concentração torna-se negativa a partir de 2006. Ressalta-se que a participação de outros rendimentos sobre a renda total no estado do Ceará não é expressiva - em torno de $5 \%$ (Tabela 6). De certo modo, isso sugere que os programas de transferência de renda do governo federal contribuíram para a redução 
da desigualdade de renda no período. No entanto, deve-se investigar esse fato de maneira mais pormenorizada.

Conforme Araújo (2009), no entanto, vários trabalhos mostram que existem muitas controvérsias quanto à eficácia dos programas de transferência de renda. Alguns autores afirmam que as transferências governamentais desencorajam os indivíduos pobres a procurarem emprego e os tornam dependentes das transferências contínuas do governo. Por outro lado, outros estudos apontam para a eficácia desses programas.

Medeiros, Brito e Soares (2007) afirmam que os programas de transferência de renda no Brasil contribuem para reduzir a pobreza e a desigualdade. Ressaltam, ainda, que não há indicação de que as transferências afetam de modo substantivo a participação das famílias no mercado de trabalho. Verificaram, inclusive, que a participação no mercado de trabalho aumenta entre os beneficiários.

Apesar de os programas de transferência de renda serem importantes para contribuir na diminuição da desigualdade de renda domiciliar per capita, deve-se levar em consideração que os dados sobre participação dos componentes de renda na renda total e as razões de concentração nas Tabelas 6 e 7 mostram que a parcela proveniente do mercado de trabalho deve ser a principal questão a ser discutida sobre a desigualdade de renda cearense. Esses resultados corroboram com Araújo (2010), que destaca também que a escolaridade é considerada a principal causa da desigualdade salarial no Brasil.

\subsection{A decomposição da mudança do índice de Gini segundo efeito-composição e efeito-concentração}

Neste item, são mostrados os resultados da decomposição para o período 2004 a 2012, em que se pode desagregar a mudança do coeficiente de Gini pelo efeito-composição (quando existe alterações nas proporções da renda) e efeito-concentração (quando há mudanças nos índices de concentração). Esse tipo de decomposição foi utilizado 
por outros autores que estudaram a distribuição de renda no Brasil nos últimos anos, tais como o estudo de Rocha et al. (2009).

Inicialmente, examina-se a decomposição no índice de Gini de 2004 a 2012. Nesse intervalo, o coeficiente de concentração do rendimento domiciliar per capita retraiu de 0,5708 para 0,5203 , ou seja, . A Tabela 8 mostra a decomposição, conforme a metodologia discutida anteriormente, diferenciando-a em efeito-composição e efeito-concentração. O efeitoconcentração total representa $84,96 \%$ de . Verifica-se a preponderância do efeito-concentração, ou seja, a diminuição da concentração de cada parcela de renda foi o principal fator para a redução da desigualdade de renda no estado do Ceará, enquanto o efeito-composição mostra-se ser, na maioria dos casos, de pouca expressividade.

Tabela 8: Decomposição da mudança no índice de Gini da distribuição do rendimento domiciliar per capita no estado do Ceará, entre 2004 e 2012:

\begin{tabular}{|c|c|c|c|}
\hline Parcela & $\begin{array}{l}\text { Efeito-composição \% } \\
\text { de ( }\end{array}$ & $\begin{array}{l}\text { Efeito-concentração } \\
\% \text { de ( }\end{array}$ & Total \% de ( \\
\hline TRA & 0,95 & 22,81 & 23,75 \\
\hline AP1 & $-1,51$ & 36,75 & 35,25 \\
\hline AP2 & 0,15 & 3,57 & 3,73 \\
\hline ALU & $-1,02$ & 0,33 & $-0,69$ \\
\hline DOA & $-2,72$ & 2,63 & $-0,09$ \\
\hline OUT & 23,18 & 14,87 & 38,04 \\
\hline Total & 19,04 & 80,96 & 100,00 \\
\hline
\end{tabular}

Fonte: elaborado a partir dos microdados da PNAD (IBGE, 2004 e 2012)

Ao analisar as informações mostradas na Tabela 8, pode-se afirmar que as parcelas que tiveram as maiores contribuições na redução da desigualdade da renda domiciliar per capita no estado do Ceará durante o período de 2004 a 2012 são os rendimentos do trabalho (TRA), as aposentadorias e pensões públicas (AP1) e outros rendimentos (OUT). 
Diversos fatores podem ter contribuído para essas mudanças, por exemplo: alterações no mercado de trabalho, crescimento do Benefício da Prestação Continuada (BPC), aumento do valor real do salário mínimo (piso das aposentadorias e pensões oficiais) e expansão dos programas sociais do governo, como o Bolsa Família. Infelizmente, a PNAD não permite desagregar a variável "outros rendimentos", que possui, entre seus diferentes componentes, as rendas provenientes dos programas de transferências de renda do governo federal. Sem dúvida, essa variável pode ser estudada com mais detalhes em outras pesquisas.

Note que a Tabela 8 mostra que a redução da desigualdade de renda durante o período de 2004 a 2012 foi consequência da redução da desigualdade de renda no trabalho (TRA), por meio do efeitoconcentração $(23,75 \%)$, enquanto a parcela outros rendimentos (OUT) foi responsável por $38,04 \%$ da redução total da desigualdade de renda no estado do Ceará.

Já as aposentadorias e pensões públicas (AP1), apesar de se manterem regressiva em todo o período, contribuíram com 35,25\% na redução do índice de Gini. Uma determinada parcela da renda pode contribuir na redução da desigualdade, mesmo sendo regressiva.

\section{Considerações finais}

O artigo analisou a desigualdade de renda no estado do Ceará e utilizou uma metodologia de decomposição do índice de Gini. Dessa forma, foi possível encontrar os componentes de renda que influenciaram a desigualdade.

A análise da redução da desigualdade permite concluir que predomina o efeito-concentração, ou seja, a diminuição da concentração de cada parcela de renda foi o principal fator para a redução da desigualdade. Enquanto isso, o efeito-composição mostra-se ser, na maioria dos casos, de pouca expressividade.

A participação da parcela da renda proveniente do trabalho (TRA) diminuiu de $70,60 \%$ para $68,20 \%$ no estado do Ceará durante o período analisado. Esse componente de renda é o que possui a maior 
participação na renda domiciliar per capita, seguido pela parcela da renda de aposentadoria e pensões oficiais (AP1), com uma média no período de aproximadamente $21 \%$.

Sobre os determinantes da redução da desigualdade de renda, pode-se atribuir que foi devido à redução da desigualdade de renda do trabalho (TRA), por meio do efeito-concentração $(22,81 \%)$ e da parcela outros rendimentos (OUT), sendo esta última responsável por 38,04\% da redução total da desigualdade de renda de 2004 a 2012. No entanto, as aposentadorias e pensões oficias (AP1), apesar de se manterem regressivas em todo o período, contribuíram com $35,25 \%$ na redução da desigualdade de renda.

Uma das principais parcelas que contribuíram para reduzir a desigualdade de renda no estado do Ceará foi o componente outros rendimentos (OUT). Conclui-se que de, 2004 a 2012, sua contribuição foi de $35,04 \%$

Conclui-se, ainda, que a parcela da renda proveniente de outros rendimentos (OUT) tinha uma pequena participação relativa, mas elevou-se a sua contribuição na renda total ao longo do período de 2004 a 2012. Os resultados mostram que essa mudança teve impactos na distribuição de renda.

Em síntese, os rendimentos do trabalho são responsáveis pela redução da desigualdade de renda no estado do Ceará de 2004 a 2011. A renda de aposentadorias e pensões públicas (AP1) também contribuiu de forma expressiva.

Por fim, destacam-se os programas de transferência de renda. No mais, a parcela proveniente do mercado de trabalho deve ser a principal questão a ser discutida sobre a desigualdade de renda cearense em termos de políticas públicas.

\section{Referências}

ARAÚJO, J. A. Pobreza, desigualdade e crescimento econômico: três ensaios em modelos de painel dinâmico. 2009. 101f. Tese (Doutorado em Economia) - Universidade Federal do Ceará, Fortaleza, 2009. 
ARAÚJO, J. A.; MORAIS, G. A. de S. Desigualdade de renda e a sua decomposição no Brasil e nas Regiões Brasileiras. In: Encontro Economia do Ceará em Debate, IX 2013. Novembro de 2013. Fortaleza/CE. Anais... Fortaleza: IPECE. 2013. Disponível em:< http:// www2.ipece.ce.gov.br/encontro/2013/lista.htm>. Acesso em: $10 \mathrm{dez}$. 2013.

ARAÚJO, R. J. Decomposição da recente queda da desigualdade da renda per capita no Brasil: uma análise a partir do índice de concentração. 2010. 114f. Dissertação (Mestrado em Economia) Universidade Federal do Rio Grande do Sul, Porto Alegre, 2010.

BARRETO, F. A. F.D.; NETO, P. M. J.; TEBALDI, E. Desigualdade de Renda e Crescimento Econômico no Nordeste Brasileiro. Revista Econômica do Nordeste, Fortaleza, v.32, n. Especial, p.842-859, nov., 2001.

BARROS, R.P.; CARVALHO, M.; FRANCO, S.; MENDONÇA, R. Determinantes da queda da desigualdade de renda no Brasil. Texto para Discussão - n. 1460, Rio de Janeiro, jan. 2010.

BARROS, R.P.; CARVALHO, M.; FRANCO, S.; MENDONÇA, R. Uma análise das principais causas da queda recente na desigualdade de renda brasileira. Econômica, Rio de Janeiro, v.8, n.1, p.117-147, jun. 2006.

BARROS, R.P.; FOGUEL,M.N., ULYSSEA, G. Sobre a Recente Queda da Desigualdade de Renda no Brasil. In: BARROS, R.P., FOGUEL, M. N., ULYSSEA, G. (org). Desigualdade de renda no Brasil: uma análise da queda recente. Brasília: IPEA, 2006, v.1. Nota Técnica.

BARROS, A. R. C. As desigualdades regionais de renda entre indivíduos no Brasil.. Brasília, DF: IPEA, abr., 2013. Disponível em: < http://www.bnb.gov.br/content/aplicacao/eventos/forumbnb2012/ docs/ sim1_mesa1_dinamica_desigualdades_regionais_brasil.pdf>. Acesso em: 16 dez. 2013. 
FOCHEZATTO, A. Estrutura da Demanda Final e Distribuição de Renda no Brasil: uma abordagem multissetorial utilizando uma matriz de contabilidade social. Revista Economia, Brasília, v.12, n.1, p.111-130, jan/abr 2011.

HOFFMANN, R. Transferências de renda e redução da desigualdade no Brasil e em cinco regiões, entre 1997 e 2005. In: BARROS, R.P.; FOGUEL, M.N.; ULYSSEA, G. (Org). Desigualdade de renda no Brasil: uma análise da queda recente. Brasília, DF: IPEA, v.2, p.17-40, 2007.

INSTITUTO DE PESQUISA ECONÔMICA APLICADA (IPEA). Comunicado do IPEA no 155 - A Década Inclusiva (2001-2011): desigualdade, pobreza e políticas de renda. Brasília, DF: IPEA, 2012. Disponível em: <http://www.ipea.gov.br/agencia/images/stories/PDFs/ comunicado/120925_comunicadodoipea155_v5.pdf>. Acesso em: 17 dez. 2013.

INSTITUTO DE PESQUISA E ESTRATÉGIA ECONÔMICA DO CEARÁ (IPECE). Informe $N^{\circ}$ 19: a evolução da desigualdade de renda entre os anos de 2000 e 2010 no Ceará e estados brasileiros. Quais foram os avanços?. Fortaleza, CE: IPECE, 2011. Disponível em:< http:// www.ipece.ce.gov.br/economia-do-ceara-em-debate/ix-encontro/ certificados/artigos/cer tificado\%20DECOMPOSICAO\%20DA\%20 DESIGUALDADE\%20DE\%20RENDA\%20NO\%20BRASIL\%20E\%20 NO\%20ESTADO\%20DO\%20CEARA.pdf>. Acesso em: 16 dez. 2013.

MARINHO, E.; LINHARES, F; CAMPELO, G. Os programas de transferências de renda do governo impactam a pobreza no Brasil? Fortaleza: UFC/CAEN, 2007. Série Ensaios sobre a pobreza, n. 12. Disponível em: <http://www.caen.ufc.br/lep.htm>. Acesso em: 09 jul. 2012.

MARQUES, A. Crescimento e desigualdade no nordeste do Brasil: 2002-2006. Revista Nexos Econômicos, v.4, n.6, p.89-115, jun.,2010. 
MEDEIROS, M.; BRITO, T. SOARES F. Programas focalizados de transferência de renda no Brasil: contribuições para o debate. Rio de Janeiro: IPEA, 2007. (Texto para Discussão, 1283).

MIRO, V. H.; SULIANO, D. C.; OLIVEIRA, J. L. Fatores determinantes para a queda da desigualdade no Ceará na última década. Fortaleza: IPECE, 2011. (Textos para discussão, n.92).

NEDER, H. D.; SILVA, J. L. M. Pobreza e Distribuição de Renda em Áreas Rurais: uma Abordagem de Inferência. Revista de Economia e Sociologia Rural, Rio de Janeiro, v.42, n.3, p.469-486, set. 2004.

PENNA, C. M.; NETO, N. T. Renda do trabalho, renda de transferências e desigualdade: uma nova proposta de investigação empírica da curva de Kuznets para o Ceará. Fortaleza: IPECE, 2009. (Textos para discussão, n.66).

PONTES, P. Os determinantes da redução da desigualdade espacial no Ceará nas últimas décadas. Fortaleza: IPECE, 2011 (Textos para discussão, n.98).

RAMOS, L. Desigualdade de Rendimentos do Trabalho no Brasil, de 1995 a 2005. In: BARROS, R. P.; FOGUEL, M. N.; ULYSSEA, G. (Org). Desigualdade de renda no Brasil: uma análise da queda recente. Brasília, DF: IPEA, v.2, p.267-284, 2007.

ROCHA, H.; MAYORGA, D.; LIMA, P.V.P.S.; MAYORGA, I. A contribuição das parcelas do rendimento mensal domiciliar para a distribuição de renda no meio rural do estado do Ceará. In: Encontro de Economia do Ceará em Debate. Fortaleza, V, 2009. Fortaleza. Anais... Fortaleza: IPECE, 2009. p. 1-17. Disponível em: <http://www.ipece.ce.gov.br/ economia-do-ceara-em-debate/>. Acesso em: 11 jun. 2013.

ROCHA, S. Pobreza no Brasil: afinal de que se trata? 3 ed. Rio de Janeiro: FGV, 2006.

SALVATO, M. A.; FERREIRA, P. C. G.; DUARTE, A. J. M. O Impacto da Escolaridade Sobre a Distribuição de Renda. Revista Estudos Econômicos, São Paulo, v.40, n.4, p.753-791, out., 2010. 
SOARES, S. S. D. Distribuição de renda no Brasil de 1976 a 2004 com ênfase no período entre 2001 e 2004. Brasília: IPEA, 2006. (Texto para Discussão. n. 1.166).

Artigo recebido em: 22/02/2014

Aprovado em: 14/10/2014 